\title{
Management of Vulval Lichen Planus
}

\author{
D.Maor $^{*}$, C.Heyes ${ }^{1}$, A.Howard ${ }^{1}$, E.Veysey ${ }^{1}$ \\ 'Department of Dermatology, Royal Women's Hospital, Parkville Victoria, Australia \\ "maor.danit@gmail.com
}

\begin{abstract}
Background: Vulval lichen planus $(V L P)$ is a debilitating, often treatment resistant condition, for which there is limited evidence-based guidance on assessment and management.
\end{abstract}

Aim: To describe current management of women with VLP in the multi-disciplinary vulval dermatology clinic at the Royal Women's Hospital (RWH), Melbourne, to compare this with reported practices in the literature and provide focus for future research needs.

Methods: A retrospective case note review was undertaken of all women with VLP attending the vulval clinic between 2009 and 2012. Data was collected regarding age of onset, symptoms, examination findings and treatments utilized.

Results: Eighty women with VLP were identified between 2009-2012. The mean age of onset was 59.8 years. Very potent topical steroids were the most commonly used first line therapy (71.3\%). The most commonly utilized agent was betamethasone diproprionate optimized-vehicle ointment $(46.3 \%$ of patients) with improvement in 16 (43.2\%). This was followed by Clobetasol propionate ointment $0.05 \%$ which was used in 8 patients (10.0\%), with improvement in 6 (75.0\%). The most frequently used systemic monotherapy was methotrexate, used in 16 patients either first or second line with improvement seen in 7 (43.8\%) with a greater proportion responding to higher doses of 10mg per week. Prednisolone was used in 7 patients, with a response noted in 3. Responders had all been commenced on a higher dose of 50mg. No consistent disease severity measures were used, clinician or patient reported.

Conclusion: This case note review describes the current practice of Dermatologists working at a subspecialty vulval dermatology unit at a tertiary referral centre. Topical corticosteroids are utilized as first line therapy, with methotrexate the most commonly used systemic agent for refractory cases. Our study also highlights the need for validated disease severity and quality of life measures. The therapeutic regimes are partially in line with published data from the UK, the Netherlands and Iowa but differ from another large Australian centre. Future research should focus on developing disease severity measures and conducting pragmatic trials comparing commonly used treatment regimes such as with Clobetasol proprionate.

\section{INTRODUCTION}

Vulval lichen planus (VLP) is an immune mediated inflammatory condition with three main clinical variants: erosive, papulosquamous and hypertrophic [1]. The disease can have a profound impact on quality of life and is often a therapeutic challenge due to treatment resistance in all variants of VLP and limited evidence-based guidance on which to base assessment and treatment decisions.

VLP is a relatively uncommon condition. The exact prevalence is unknown although in the UK, VLP accounts for approximately $1 \%$ of referrals to dermatologists [2]. It typically occurs in women aged 50-60 years who may present with constant or intermittent pain, burning, pruritus and/or dyspareunia. The examination findings are variable but may include erythema, erosions, a white lacey pattern, hypertrophic areas and scarring with architectural distortion [3].

The most common form of VLP is the erosive variant, which presents as glassy, brightly erythematous erosions associated with white reticulate striae known as Wickham's striae $[1,4,5]$. Erosive vulval LP (EVLP) is a chronic inflammatory condition [6] which may cause severe scarring and tissue destruction resulting in urinary and sexual impairment.

Although the pathogenesis of LP remains unclear, there is some evidence supporting a T-lymphocyte response to autoantigens [7]. This in turn results in apoptosis of epithelial cells and chronic inflammation. Hence, most therapies reported to improve LP have immune modulatory or immune 


\section{D.Maor et al.}

suppressive effects [8]. An HLA-associated genetic susceptibility has been reported [9]. Chronic liver disease, particularly that due to Hepatitis $\mathrm{C}$ infection, may be associated with lichen planus in certain parts of the world such as the Mediterranean basin and Japan but has not been found in the UK [10].

The lack of standardised outcome measures has been highlighted by a systematic review [3], which greatly hinders meaningful research into this and other vulval diseases.

The aims of this study were to describe current management of women with VLP in the multidisciplinary vulval dermatology clinic at the RWH, Melbourne, to compare this with reported practices from the literature and provide focus for future research needs.

\section{METHODS}

A retrospective case note review of all patients diagnosed with VLP (all variants) between 2009-2012 was undertaken. Patients were identified via a prospectively maintained database of all women attending the vulval clinic at the RWH. Ethics approval was sought but not considered necessary by the hospital ethics committee. All patients with a dermatologist-determined diagnosis of VLP were included. Data collected comprised age at presentation, duration of symptoms, symptoms, examination findings, histology and treatment utilized.

Patients were categorized as either responders (treatment remained unchanged or downgraded) or non-responders (second or third-line treatment required). Speculum examination was not routinely performed so vaginal disease could not be commented upon.

\section{RESULTS}

Eighty women were identified with a clinical diagnosis of VLP. The mean age at presentation was 59.8 years (range 19-81 years). The median duration of symptoms prior to diagnosis was 2.5 years. Onset of disease was premenopausal in 6 patients (7.5\%), perimenopausal in 8 (10\%), and postmenopausal in $66(82.5 \%)$.

Table 1 details the frequency of presenting symptoms and examination findings. No standardized severity scores, either patient or clinician reported were used. Two out of 70 patients, who reported pain, were assessed using a pain score. There were no patients who had evidence of dysplasia or squamous cell carcinoma.

Table 1: Frequency of presenting symptoms to the vulval clinic

\begin{tabular}{|c|c|}
\hline & FREQUENCY OF SYMPTOMS \\
\hline Symptom & Patients, No. (\%) \\
\hline Pain & $70(88)$ \\
\hline Pruritus & $66(83)$ \\
\hline Dyspareunia & $37(46)$ \\
\hline Dysuria & $34(43)$ \\
\hline Bleeding & $12(15)$ \\
\hline Vaginal discharge & $14(18)$ \\
\hline Asymptomatic & 0 \\
\hline
\end{tabular}

A biopsy was performed in 57 (71\%) patients. Of these, 48 confirmed lichen planus, and 9 were considered non-diagnostic. The remainders were not biopsied as the clinical picture was strongly suggestive of VLP or the patient declined biopsy.

\subsection{Treatment}

\subsubsection{First-line therapy}

First-line therapy and response are detailed in Table 2. Monotherapy with a topical agent was used in $62(78 \%)$ patients, with 60 of these treated with a topical steroid. Fifteen patients $(19 \%)$ were commenced on dual topical first-line therapy, with a topical steroid in combination with either clindamycin or clotrimazole. Of the remaining patients, 2 (2.5\%) were commenced on a systemic agent methotrexate alone and $1(1.25 \%)$ was commenced on prednisolone.

Of those 60 patients on topical steroid first-line monotherapy, 8 (10\%) received clobetasol proprionate ointment $0.05 \%, 5$ (63\%) of which responded; 37 (46.25\%) betamethasone dipropionate in optimizedvehicle ointment, $16(43 \%)$ of which responded; 12 (15\%) methylprednisolone aceponate ointment, 
none of which responded and 3 patients (3.75\%) were commenced on hydrocortisone acetate $5 \%$ foam (administered topically to the vulva), none of which responded. Average duration of first-line therapy before determining response was 15.4 weeks and overall, 26 patients (32\%) were graded as having a good response. All patients were advised to include good vulval skin care in the management of their VLP, which included use of a soap-free wash and wearing cotton underwear.

Table 2. Efficacy of First-line therapy

\begin{tabular}{|l|c|ccc|}
\hline & No. (\%) & \multicolumn{3}{|c|}{ Response, No (\%) } \\
& Prescribed & Good & Moderate & Poor \\
\hline Treatment & & & & \\
Corticosteroid monotherapy & $37(46.25)$ & $16(43.24)$ & $4(10.81)$ & $17(45.95)$ \\
Betamethasone diproprionate ointment 0.05\% & $8(10)$ & $5(62.5)$ & $1(12.5)$ & $2(25)$ \\
Clobetasolproprionate ointment 0.05\% & $10(12.5)$ & 0 & 0 & $10(100)$ \\
Methylprednisolone aceponate ointment 0.1\% & $2(2.5)$ & 0 & 0 & $2(100)$ \\
Methylprednisolone aceponate cream & $3(3.75)$ & 0 & 0 & $3(100)$ \\
Hydrocortisone acetate 5\% foam & & & & \\
Other topical therapies & $1(1.25)$ & 0 & 0 & $1(100)$ \\
Clindamycin & $1(1.25)$ & 0 & $1(100)$ & 0 \\
Clindamycin and clotrimazole & & & & \\
Dual topical therapy & $7(8.75)$ & $2(28.57)$ & $1(14.29)$ & $4(57.14)$ \\
Betamethasone diproprionate ointment and clindamycin & $1(1.25)$ & 0 & 0 & $1(100)$ \\
Betamethasone diproprionate ointment and clotrimazole & $3(3.75)$ & $1(33.33)$ & $1(33.33)$ & $1(33.33)$ \\
Hydrocortisone foam and topical tacrolimus 0.1\% ointment & $2(2.5)$ & 0 & $1(50)$ & $1(50)$ \\
Betamethasone diproprionate cream, clindamycin and oestrodial & $2(2.5)$ & $1(50)$ & $1(50)$ & 0 \\
Clobetasolproprionate ointment and clindamycin & & & & \\
Systemic & $1(1.25)$ & $1(100)$ & 0 & 0 \\
Methotrexate (5-7.5mg) & $1(1.25)$ & 0 & 0 & $1(100)$ \\
Prednisolone & & & & \\
Topical steroid and systemic & $1(1.25)$ & 0 & $1(100)$ & 0 \\
Betamethasone diproprionate ointment and methotrexate & $\mathbf{8 0}$ & $\mathbf{2 6}$ & $\mathbf{1 1}$ & $\mathbf{4 3}$ \\
\hline TOTAL & & &
\end{tabular}

\subsubsection{Second-line therapy}

Second-line therapy and response are detailed in Table 3. Fifty-four patients $(67.5 \%)$ required second line treatment. Twenty (37\%) responded and $34(62.9 \%)$ required third-line treatment (Table 3) after an average of 15.3 weeks. Systemic agents were used in 24 patients and included methotrexate (15), prednisolone (7), hydroxychloroquine (1), azathioprine (1) (mostly used in conjunction with a TCS) and one patient was commenced on both methotrexate and prednisolone in combination with a topical steroid. Six patients were commenced on a higher dose of $10 \mathrm{mg}$ methotrexate and all responded in comparison to the 8 patients who were commenced on a lower dose of 5-7.5mg who required treatment upgrade.

Six patients were treated with prednisolone between doses 5mg to 50mg. Amitriptyline (12) and gabapentin (1) were used on occasion as adjunctive treatments with good outcomes.

Table 3. Efficacy of Second--line therapy

\begin{tabular}{|l|c|ccc|}
\hline \multicolumn{1}{|c|}{ Treatment } & \multicolumn{3}{c|}{ No. (\%) } & \multicolumn{3}{c|}{ Response, No (\%) } \\
Prescribed & Good & Moderate & Poor \\
\hline Systemic monotherapy & & & & \\
Methotrexate (5-7.5mg) & $10(18.51)$ & $4(40)$ & $5(50)$ & $1(10)$ \\
Prednisolone(5-50mg) & $5(9.25)$ & $2(40)$ & $1(20)$ & $2(40)$ \\
Hydroxychloroquine & $1(1.85)$ & 0 & $1(100)$ & 0 \\
Azathioprine & $1(1.85)$ & 0 & 0 & $1(100)$ \\
Topical and systemic therapy & $4(7.4)$ & $2(50)$ & $2(50)$ & 0 \\
TCS and methotrexate & $1(1.85)$ & $1(100)$ & 0 & 0 \\
TCS andprednisolone & $1(1.85)$ & 0 & 0 & $1(100)$ \\
TCS and methotrexate and prednisolone & $1(1.85)$ & $1(100)$ & 0 & 0 \\
TCS andfluconazole & & & & \\
Combined topical & & & & \\
\hline
\end{tabular}




\begin{tabular}{|c|c|c|c|c|}
\hline Betamethasone diproprionate ointment and clindamycin & $3(5.55)$ & $2(66.6)$ & $1(33.33)$ & 0 \\
\hline Betamethasone diproprionate ointment and oestradiol & $1(1.85)$ & $1(100)$ & 0 & 0 \\
\hline Methylprednisolone aceponate ointment and clindamycin & $2(3.7)$ & 0 & 0 & $2(100)$ \\
\hline Pimecrolimus and clindamycin & $1(1.85)$ & $1(100)$ & 0 & 0 \\
\hline \multicolumn{5}{|l|}{ Other topical } \\
\hline Clindamycin & $2(3.7)$ & 0 & $1(50)$ & $1(50)$ \\
\hline Pimecrolimus & $1(1.85)$ & 0 & $1(100)$ & 0 \\
\hline \multicolumn{5}{|l|}{ Topical corticosteroids } \\
\hline Clobetasolproprionate ointment $0.05 \%$ & $7(12.96)$ & $2(28.57)$ & $4(57.14)$ & $1(14.29)$ \\
\hline Methylprednisolone & $4(7.4)$ & $2(50)$ & $1(25)$ & $1(25)$ \\
\hline Betamethasone diproprionate ointment & $9(16.66)$ & $2(22.22)$ & $4(44.44)$ & $3(33.33)$ \\
\hline TOTAL & 54 & 20 & 21 & 13 \\
\hline
\end{tabular}

\section{DISCUSSION}

This retrospective case note review examines the management of patients with VLP presenting to the multidisciplinary vulval clinic at the Royal Women's Hospital in Melbourne, Australia. It provides insight into current Australian practice and allows comparison with other international centres.

The mean age of presentation to the RWH of 59.8 years is consistent with current literature [3, 6]. A UK audit of treatment used in 172 patients with VLP established that a very potent topical steroid (clobetasol diproprionate ointment) was used as first line therapy in $75 \%$ of cases with a $66 \%$ response rate [3]. Other studies have reported a $75 \%$ improvement in response to a very potent topical steroid, mostly clobetasol proprionate ointment [3]. A retrospective clinical study by Santegoets et al. in the Netherlands reported that of 95 patients treated with topical steroids, $70 \%$ showed a slight to moderate improvement with no improvement at all in 30\% [11]. A majority of their patients $(67.4 \%)$ were initially treated with Clobetasol propionate $0.05 \%$ ointment twice daily for a month before tapering to a maintenance treatment [11]. None of their patients were treated with systemic medication [11]. Kennedy and Galask in the U.S performed a retrospective review, which concluded that treatment with TCS was employed at the lowest potency possible and when response was adequate, treatment was tapered off [12]. They also used topical immune suppressant therapy (tacrolimus and pimecrolimus), which did not result in a better outcome than the other treatments employed (typically topical steroids) [12].

In Australia, Clobetasol proprionate ointment is not available as a proprietary agent; therefore Betamethasone dipropionate OV is used as an alternative and considered to be close to Clobetasol dipropionate $0.05 \%$ in potency. However, in our study $62.5 \%$ responded to topical Clobetasol dipropionate, in keeping with the published literature and only $43.2 \%$ to Betamethasone dipropionate OV as first line therapy.

Another retrospective audit from a large centre in Sydney [13] of 131 patients with VLP concluded that oral prednisolone alone at a mean dose of $17 \mathrm{mg}$ or $5 \mathrm{mg}$, as an adjunct to TCS was effective, safe and well tolerated [13]. In our study, only one patient was commenced on oral prednisolone (5mg) as first-line treatment with no response. $11.1 \%(6 / 54)$ were commenced on oral prednisolone alone as second-line treatment with the dose tapered as symptoms improved. Three patients were commenced on a higher dose of $50 \mathrm{mg}$ and had better response than the three who were on $20 \mathrm{mg}$ and $5 \mathrm{mg}$ Prednisolone.

The UK audit by Simpson et al. used 16 different systemic agents when other first-line treatments failed to provide adequate disease control [3]. Prednisolone (13/37) and hydroxychloroquine (8/37) were the most frequently used initial systemic agents after failure of topical treatment. Hydroxychloroquine was used longer-term to maintain disease control [3]. In our study, hydroxychloroquine was only used in 1 patient as second line therapy and was not effective. Fisher and Bradford [13] report on the successful use of prednisolone and methotrexate as first line therapy, which is partially supported by our findings. Systemic therapy should be considered in cases where permanent scarring is probable or when topical therapy has failed to halt the progression of the disease. Currently, there is insufficient evidence to recommend a particular systemic agent [5].

A randomized control trial was recently published on vulvovaginal photodynamic therapy vs. topical corticosteroids in erosive lichen planus [14]. Forty women were randomized to one session PDT or 
daily applications of clobetasol proprionate $0.05 \%$ ointment (or optional hydrocortisone acetate $1 \%$ foam) and after 6 weeks all patients were allowed to use topical corticosteroids as needed. The reduction after 6 weeks in clinical scores and in pain visual analogue scale scores was measured. Patients in the PDT group reported significantly less topical corticosteroids and the study concluded that vulvovaginal PDT seems to be an effective and safe treatment for erosive lichen planus.

The RWH does not routinely utilise patient reported outcome measures such as impact of disease on quality of life or a validated disease severity score. Clinical assessment was mostly documented via a schematic diagram of clinical features and a global severity score (mild, moderate, severe which is in line with other practice). A UK audit on randomized controlled trials for vulval skin disorders concluded that there was a lack of standardized outcome measures [3] and assessment mostly consisted of a diagrammatic representation of clinical features (87\%), and/or global measures such as mild/moderate/severe as determined by the treating clinician [15].

The major limitation of this case note review was the lack of documented severity measures.

This study carries the inherent limitations of a retrospective case file review. Certain variables that may have influenced the findings were not consistently documented and therefore could not be measured, namely the presence of vaginal, oral or cutaneous LP, other co-existing vulval disorders for example atrophic vulvo-vaginitis and concomitant medical therapy (including oestrogen replacement). The retrospective grading of severity and level of improvement will have inevitably misclassified some patients and skewed the findings. However the findings are consistent with those of other studies and ultimately are meant to inform future investigation rather than provide empirical evidence.

In conclusion, women with VLP presenting to the RWH vulval clinic are treated with a potent topical steroid as first line and typically either methotrexate or prednisolone as second line, which is in line with published reports from other centres. However, there is evidence that treating early with low dose prednisolone or methotrexate in conjunction with a TCS may improve outcomes earlier and this requires further investigation. Future research should focus on developing severity measures for women with vulval disease and establishing efficacy for commonly used treatment regimes.

\section{What's already known about this topic?}

- VLP is an immune mediated inflammatory condition that is often resistant to treatment.

- There is a paucity of evidence on which to base treatment decisions.

- There are no disease specific quality of life or severity measures.

What does this study add?

- Supports the use of ultra-potent topical steroids as first line treatment

- Provides limited evidence for the use of methotrexate as a second line therapy

- Highlights the heterogeneity in the assessment and management of VLP.

Legend:

1. Table 1: Frequency of presenting symptoms to the vulval clinic

2. Table 2: Efficacy of First-line therapy

3. Table 3: Efficacy of Second-line therapy

\section{REFERENCES}

1. Moyal-Barracco M, Edwards L. Diagnosis and therapy of anogenital lichen planus. Dermatologic therapy. 2004; 17(1):38-46.

2. Bolognia J, Bylaws Task F. Proposed revisions to bylaws of the International Society of Dermatology. International journal of dermatology. 2009 Feb; 48(2):196-200.

3. Simpson RC, Littlewood SM, Cooper SM et al. Real-life experience of managing vulval erosive lichen planus: a case-based review and U.K. multicentre case note audit. The British journal of dermatology. 2012 Jul; 167(1):85-91.

4. Lewis FM. Vulval lichen planus. The British journal of dermatology. 1998 Apr; 138(4): 569-75.

5. Goldstein AT, Metz A. Vulvar lichen planus. Clinical obstetrics and gynecology. 2005 Dec; 48 (4):818-23. 


\section{D.Maor et al.}

6. Cooper SM, Wojnarowska F. Influence of treatment of erosive lichen planus of the vulva on its prognosis. Archives of dermatology. 2006 Mar; 142(3): 289-94.

7. Simpson RC, Thomas KS, Murphy R. Vulval erosive lichen planus: a qualitative investigation of U.K. clinician views and principles of management. The British journal of dermatology. $2013 \mathrm{Jul}$; 169(1):226-7.

8. Cheng S, Kirtschig G, Cooper $\mathrm{S}$ et al. Interventions for erosive lichen planus affecting mucosal sites. Cochrane database of systematic reviews. 2012; 2: CD008092.

9. Johnson RAF, Thomas B. Fitzpatrick's colour atlas and synopsis of clinical dermatology. Sixth edition.: McGraw-Hill; 2009.

10. Cooper SM, Kirtschig G, Jeffery KJ et al. No association between hepatitis B or C viruses and vulval lichen planus in a UK population. BJOG: an international journal of obstetrics and gynaecology. 2004 Mar; 111(3):271-3.

11. Santegoets L, Helmerhorst T, van der Meijden W. A retrospective study of 96 women with a clinical diagnosis of genital lichen planus. J Low Genit Tract Dis 2010; 14: 323-8.

12. Kennedy CM, Galask RP. Erosive vulvar lichen planus: retrospective review of characteristics and outcomes in 113 patients seen in a vulvar specialty clinic. J Reprod Med 2007; 52: 43-7.

13. Bradford J, Fischer G. Management of Vulvovaginal Lichen Planus: A New Approach. J Low Genit Tract Disease. 2013 Jan; 17(1):38-32.

14. Helgesen, A. L, Warloe, T, Pripp, A. $\mathrm{H}$ et al. Vulvovaginal photodynamic therapy vs. topical corticosteroids in genital erosive lichen planus: a randomised control trial.

15. Simpson RC, Thomas KS, Murphy R. Outcome measures for vulval skin conditions: a systematic review of randomized controlled trials. The British journal of dermatology. 2013 Sep; 169(3):494501. 